\title{
Manipulation of Rotational States With Varying Pulse Parameter: Achieving High Molecular Alignment and Orientation for $\mathrm{CH} 3 \mathrm{~F}$
}

Kalyani Chordiya

ELI-HU Research and Development Non-Profit

Irén Simkó

Eötvös Loránd University

Tamás Szidarovszky

Eötvös Loránd University

Mousumi Upadhyay Kahaly ( $\square$ Mousumi.UpadhyayKahaly@eli-alps.hu )

ELI-HU Research and Development Non-Profit

Research Article

Keywords:

Posted Date: January 24th, 2022

DOI: https://doi.org/10.21203/rs.3.rs-1254013/v1

License: (c) (i) This work is licensed under a Creative Commons Attribution 4.0 International License.

Read Full License 


\title{
Manipulation of Rotational States With Varying Pulse Parameter: Achieving High Molecular Alignment and Orientation for $\mathrm{CH}_{3} \mathrm{~F}$
}

\author{
Kalyani Chordiyaa ${ }^{1,2,+}$, Irén Simkó ${ }^{3,+}$, Tamás Szidarovszky ${ }^{3, *}$, and Mousumi Upadhyay \\ Kahaly ${ }^{1,2, *}$
}

${ }^{1}$ ELI-ALPS, ELI-HU Non-Profit Ltd., Szeged, Hungary

${ }^{2}$ Institute of Physics, University of Szeged, Dóm tér 9, H-6720 Szeged, Hungary

${ }^{3}$ Institute of Chemistry, ELTE Eötvös Loránd University, Budapest, Hungary

*tamas.janos.szidarovszky@ttk.elte.hu, Mousumi.UpadhyayKahaly@eli-alps.hu

+these authors contributed equally to this work

\begin{abstract}
Increasing interest in the fields of high-harmonics generation, laser-induced chemical reactions, and molecular imaging of gaseous targets demands high molecular alignment and orientation (AnO). In this work, we examine the critical role of different pulse parameters on the field-free AnO dynamics of the $\mathrm{CH}_{3} \mathrm{~F}$ molecule, and identify experimentally feasible optical and THz range laser parameters that ensure maximal $\mathrm{AnO}$ for such molecules. Herein, apart from rotational temperature, we investigate effects of varying pulse parameters such as, pulse duration, intensity, frequency, and carrier envelop phase (CEP). By analyzing the interplay between laser pulse parameters and the resulting rotational population distributions, the origin of specific AnO dynamics was addressed. We could identify two qualitatively different AnO behaviors and revealed their connection with the pulse parameters and the population of excited rotational states. We report here the highest alignment of $\left\langle\cos ^{2} \theta\right\rangle=0.843$ and orientation of $\langle\cos (\theta)\rangle=0.886$ for $\mathrm{CH}_{3} \mathrm{~F}$ molecule at $2 \mathrm{~K}$ using single pulse. Our study should be useful to understand different aspects of laser-induced unidirectional rotation in heteronuclear molecules, and in understanding routes to tune/enhance AnO in laboratory conditions for advanced applications.
\end{abstract}

\section{Introduction}

Molecular alignment and orientation (AnO) is essential in the fields of ultrafast science, molecular imaging, time-dependent spectroscopy and detailed interrogation of molecular dynamics ${ }^{1}$. Laser-induced field-free or sudden molecular alignment of gas-phase molecules can result in a highly peaked angular distribution of the rotational wavepacket ${ }^{2}$, and it plays an important role in improving the output signal quality of studies that are sensitive to the angle between the molecule and the direction of polarization of the laser field, ${ }^{3,4}$ such as high harmonic generation ${ }^{5}$, strong field ionization ${ }^{6}$, laser induced reactions ${ }^{7}$, time dependent spectroscopy ${ }^{8}$, attosecond pulse shaping ${ }^{9}$, and molecular orbital tomography. ${ }^{10}$ Many theoretical and experimental efforts $^{1-13}$ are reported towards understanding and controlling the non-adiabatic and adiabatic AnO dynamics of molecules with different symmetry. To list a few, the intermediate alignment regime, i.e., the duration of the laser pulse in between the adiabatic and sudden limits, was investigated by Ortigoso et al. ${ }^{14}$, Torres et al. ${ }^{3}$ and Seideman et al. ${ }^{15}$. Alignment dynamics of different systems with varying individual pulse parameter such as, effect of pulse intensity was studied for the iodobenzene molecule by Lotte Holmegaard et al. ${ }^{16}$, and for the $\mathrm{O}_{2}$ and $\mathrm{N}_{2}$ molecules by P. Peng ${ }^{17}$. Jérémy Bert et al. ${ }^{18}$ illustrated the time-resolved rotational dynamics in $\mathrm{CO}_{2}$ gas after excitation with a single linearly polarized laser pulse, and unidirectional molecular rotation induced by a pulse with twisted polarization. Kenta Mizuse et al. ${ }^{19}$ reported high-precision time-resolved Coulomb explosion imaging of the rotational wave packets induced by a polarization-skewed double-pulse to investigate the creation process and dynamics of the packets in $\mathrm{N}_{2}$ molecules. Jing-Song Liu et al. ${ }^{20}$ investigated effects of the characteristics of molecules and external fields on field-free molecular orientation, through the comparison of $\mathrm{HBr}$ with $\mathrm{LiH}$ driven by the combination of a two-color laser pulse and a time-delayed $\mathrm{THz}$ laser pulse.

Interplay between pulse parameters and rotational dynamics have been studied theoretically and experimentally using OCS molecules in $\mathrm{THz}$ fields ${ }^{21}$. Juan et al. reported that the rotational revival in single pulse case can be maintained for forty times longer than the duration of the pulse ${ }^{22}$. Fleischer et al. reported the selective excitation of rotational states in isotoposlogues to isolate the effect of a desired isotope ${ }^{23}$. Using phase-shaped femtosecond ionizing laser pulses Hertz et al. reported excitation of only odd $J$ states of the $\mathrm{O}_{2}$ molecule, and achieved alignment of 0.86 at $60 \mathrm{~K}^{24}$. Experimental investigation 
of the nonadiabatic rotational excitation for ground state symmetric-top molecules, by two intense nonresonant ultrafast laser fields, leads to excitation of $\Delta J=2$ states if $K=0$ while $\Delta J=1$ and $\Delta J=2$ if $K>0^{25}$. Hirokazu et al. calculated the time evolution of the rotational-state distribution for NO molecules at varying pump intensities ${ }^{26}$. However, a comprehensive study on the interplay between excitation of different rotational states with varying, experimentally feasible non-resonant or THz laser pulse parameters is missing in the literature, to the best of our knowledge.

This work aims to reveal how the different pulse parameters, distributions of populations in the excited rotational states, and resulting AnO dynamics relate to each other, and should help one in analysing and tuning the maximal laser-induced alignment and orientation. Analysis protocols similar to those prescribed in this work can be applied for other symmetric top molecules, as well. In this theoretical work we utilize experimentally feasible $800 \mathrm{~nm}^{27,28}$ wavelength and THz frequency pulse parameters $^{29,30}$ and the methyl fluoride $\left(\mathrm{CH}_{3} \mathrm{~F}\right)$ as a symmetric top prototype molecule. The tuning range of experimentally feasible pulse parameters are summarized in Table.1.

\begin{tabular}{|c|c|}
\hline Parameters at the laser output & Tuning range \\
\hline Peak power (800nm) & $0.1-100 \mathrm{TW} / \mathrm{cm}^{2}$ \\
Full width half maxima $(800 \mathrm{~nm})$ & $12 \mathrm{fs}-200 \mathrm{fs}$ \\
\hline Useful Spectral coverage $(\mathrm{THz})$ & $0.1-2.5 \mathrm{THz}$ \\
Peak power $(\mathrm{THz})$ & $\sim 6 \times 10^{-5} \mathrm{TW} / \mathrm{cm}^{2}-1 \mathrm{TW} / \mathrm{cm}^{2}$ \\
\hline
\end{tabular}

Table 1. Summarizing experimentally possible tuning range of pulse parameters for $800 \mathrm{~nm}^{27,28}$ and $\mathrm{THz}_{\mathrm{pulses}}^{29,30}$

\section{Results and Discussion}

$\mathrm{CH}_{3} \mathrm{~F}$ is a prolate symmetric top molecule with rotational constants $B_{z}(A)>B_{y}(B)=B_{x}(C)$. The simulated molecular parameters show reasonable match with the experimentally reported values (given in parenthesis): $B_{x}=B_{y}=0.829 \mathrm{~cm}^{-1}$ $\left(0.852 \mathrm{~cm}^{-131}\right)$ and $B_{z}$ is $5.089 \mathrm{~cm}^{-1}\left(5.182 \mathrm{~cm}^{-131}\right)$, dipole moment $\left(\mu_{z}\right)$ of $1.894 \mathrm{D}\left(1.850 \mathrm{D}^{32}\right)$, polarizability $\alpha_{\|}$as 2.524 $\AA^{3}$ and $\alpha_{\perp}$ as $2.296 \AA^{3}$. The computed molecular parameters can be improved further with higher level of theory, and using vibrational ground state geometry ${ }^{33} \cdot \mathrm{CH}_{3} \mathrm{~F}$ is a symmetric top molecule, therefore, the rotational eigenfunctions transform as the irreducible representations (irreps) of $\mathrm{D}_{\infty}{ }^{34}$. The nuclear spin statistical weights ${ }^{34}$ (NSSW) corresponding to each irrep are as follows: $\mathrm{NSSW}^{\Sigma^{+}}=2, \mathrm{NSSW}^{\Sigma^{-}}=2, \mathrm{NSSW}^{E_{1}}=1, \mathrm{NSSW}_{2}=1$, and $\mathrm{NSSW}_{3}=2$.

\section{Effect of Varying Temperature and Optical Pulse Parameters}

\begin{tabular}{|c|c|c|c|}
\hline Figure 1 & $\begin{array}{c}\text { FWHM } \\
\text { (fs) }\end{array}$ & $\begin{array}{c}\text { Intensity } \\
\left(\mathrm{TW} / \mathrm{cm}^{2}\right)\end{array}$ & $\begin{array}{c}\text { Temperature } \\
\text { (K) }\end{array}$ \\
\hline $\begin{array}{c}\text { (a,d) Temperature } \\
\text { (b,e) FWHM }\end{array}$ & 100 & 100 & 0 to $300^{*}$ \\
(c,f) Intensity & 10 to $700 *$ & 100 & 2 \\
1 to $100 *$ & 2 \\
\hline
\end{tabular}

Table 2. $800 \mathrm{~nm}$ pulse parameters used to simulate alignment of $\mathrm{CH}_{3} \mathrm{~F}$ molecule shown in panels of Fig. 1. *Varying pulse parameters for $800 \mathrm{~nm}$ pulse. Varying means only one parameter was changed, the others were kept fixed in the simulations.

In the following subsection we will discuss the effects of varying temperature and pulse parameters (see Table 2) for an optical pulse $(800 \mathrm{~nm})$. During laser-induced rotational alignment (LIRA), the $\mathrm{CH}_{3} \mathrm{~F}$ is assumed to be in a thermal ensemble at a rotational temperature $T$. The initial distribution of rotational levels is given by the Boltzmann distribution and the nuclear spin statistical weight ${ }^{34}$ of the rotational levels. When the molecular ensemble is excited with a laser pulse, the time-dependent alignment can be explained as the averaged effect of the rotational wave packets formed from each Boltzmann-weighted initial rotational state. At lower $T$ the contribution from higher energy $|J, K, M\rangle$ initial states is negligible and hence, highest alignment could be achieved at $0 \mathrm{~K}$. On exciting $\mathrm{CH}_{3} \mathrm{~F}$ at different rotational temperatures, we observe the alignment curves as given in Fig.1(a)) and the corresponding maximal alignments in Fig.1(d). At $T=0 \mathrm{~K}$ the highest achieved rotational alignment is $\left\langle\cos ^{2}(\theta)\right\rangle=0.906$, which decreases with increase in temperature, $\left\langle\cos ^{2}(\theta)\right\rangle=0.8$ at $2 \mathrm{~K}$ and $\left\langle\cos ^{2}(\theta)\right\rangle=\sim 0.5$ at $20 \mathrm{~K}$ temperature (Fig. 1(a and d)). LIRA at $0 \mathrm{~K}$ (Fig. 1(a)) shows that as the pulse hits the molecules, a sharp rise in alignment is observed whereas, the transients are observed as the manifestations of half and full revivals $\left(\tau_{\text {rev }}\right)$ where, $\tau_{\text {rev }}=$ $1 /\left(2 B_{x} c\right)=1 /\left(2 B_{y} c\right)=20 \mathrm{ps}(c$ is the speed of light). The corresponding population distribution (PD) for $|J, K, 0\rangle$ states, summed 
(a) Temperature



(d) Temperature

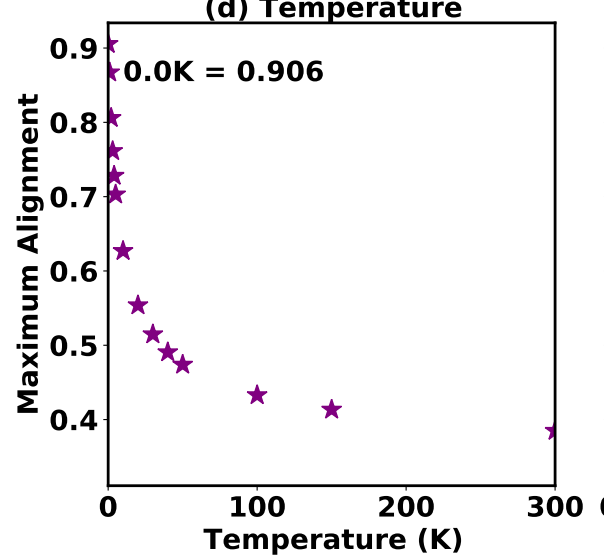

(b) FWHM

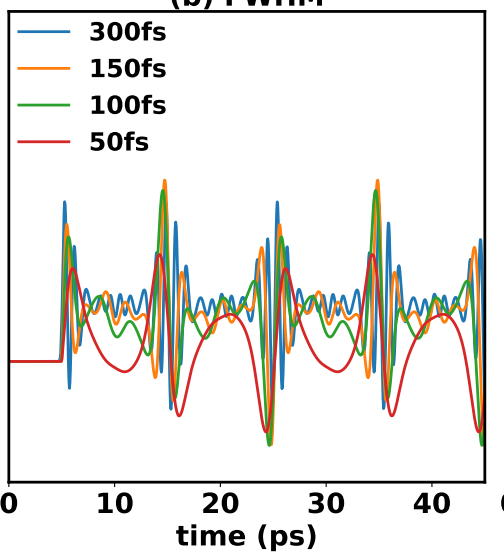

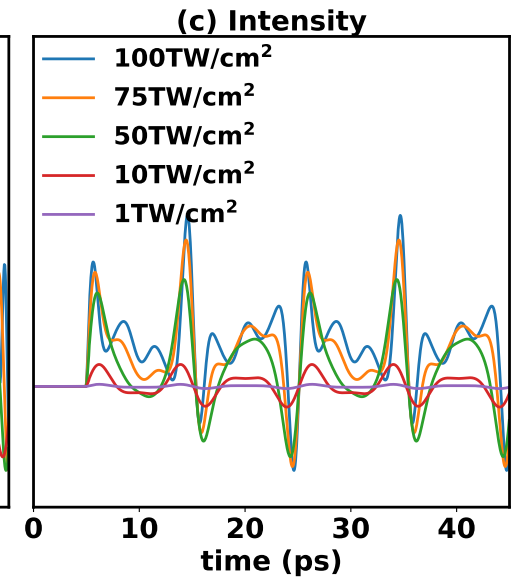

(e) FWHM

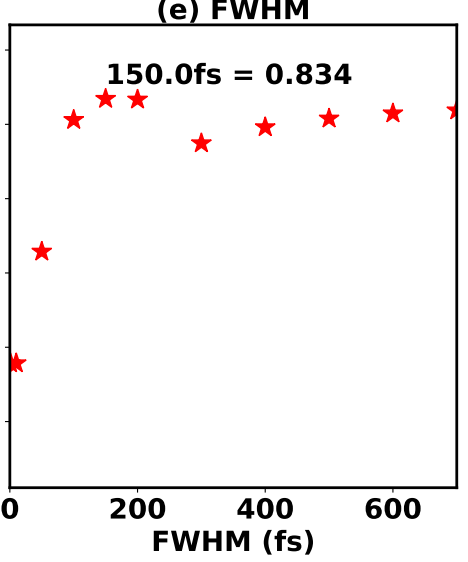

(f) Intensity

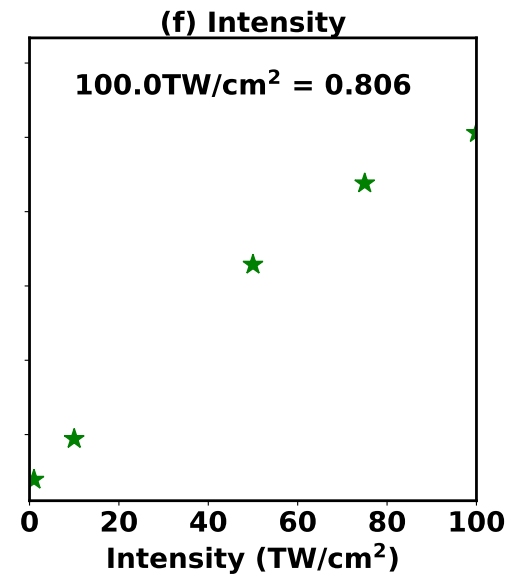

Figure 1. Effect of varying (a) Temperature, (b) FWHM and (c) Intensity on alignment with 800 nm pulse. Maximum alignment of $\mathrm{CH}_{3} \mathrm{~F}$ achieved with varying (d) Temperature, (e) FWHM and (f) Intensity in range as given in Table. 2

over $K$, is given in Fig. 2(a), the heatmap shows excitation of even $J$ states (for $M=0$ ) and gives $|2, K, 0\rangle$ as the highest populated state. On increasing the value of $T$, higher-lying $|J, K, M\rangle$ states (along with a mixture of lower even and odd $J$ states) are populated (for detailed discussion see SI) and consequently, the degree of alignment decreases (see Fig. 1(d)). All the rotational revivals for varying temperature are observed to be of conventional type, that is, the revivals are distinctly observed and are usually separated by plateaus. Note that for diatomic and polyatomic linear molecules, such as $\mathrm{N}_{2}, \mathrm{O}_{2}, \mathrm{CO}_{2}, \mathrm{OCS}_{2}$ and for the nonlinear polyatomics $\mathrm{C}_{2} \mathrm{H}_{4}$ and iodobenzene, the highest laser-induced field-free alignment of $\sim 0.5$ is achieved at room temperature by using two or multiple pulses ${ }^{35}$, which is experimentally more challenging than employing a single pulse. In this study we report achieving high alignment for $\mathrm{CH}_{3} \mathrm{~F}$ using a single non-resonant laser pulse at $2 \mathrm{~K}$. With the current advancement in molecule cooling setups, temperature as low as $1 \mathrm{~K}$ could be achieved ${ }^{36}$ using high pressure supersonic pulsed valve $^{37}$ and helium nanodroplets ${ }^{38}$. Hence, in the further study on the effect of different laser parameters on AnO, we will use $T=2 \mathrm{~K}$.

To demonstrate the effect of pulse duration on molecular alignment we increase the full width at half maximum (FWHM) of the $800 \mathrm{~nm}$ pulse from $10 \mathrm{fs}$ to $700 \mathrm{fs}$ (see Fig. 1(b and e)). The LIRA observed in Fig. 1(b) are of the conventional type for FWHM=50 fs and the corresponding PD heatmaps (see SI Fig. S2 and Fig. 2(b)) show that the excitation is mostly localized on a few states near a specific $J$ value, which increases with FWHM. However, for FWHM $\leq 150$ fs the alignment curves show quarter revival, on subsequent increase of FWHM $>150$ fs the revival evolves to an unconventional or anomalous behavior, where oscillations appear on the plateaus connecting the distinct revivals (see revival for FWHM=300 fs). Further analysis through the PD heatmaps (see SI Fig. S2) and the time-dependent population analysis (see SI Fig. S3) explains that longer FWHM allows for larger $J$ values to be populated, but also favors light-induced de-excitation, which leads to a broader distribution of $J$ states in the wave packet. In Fig. 1(e) we see the alignment increasing with the FWHM from 10 fs to 150 fs, 

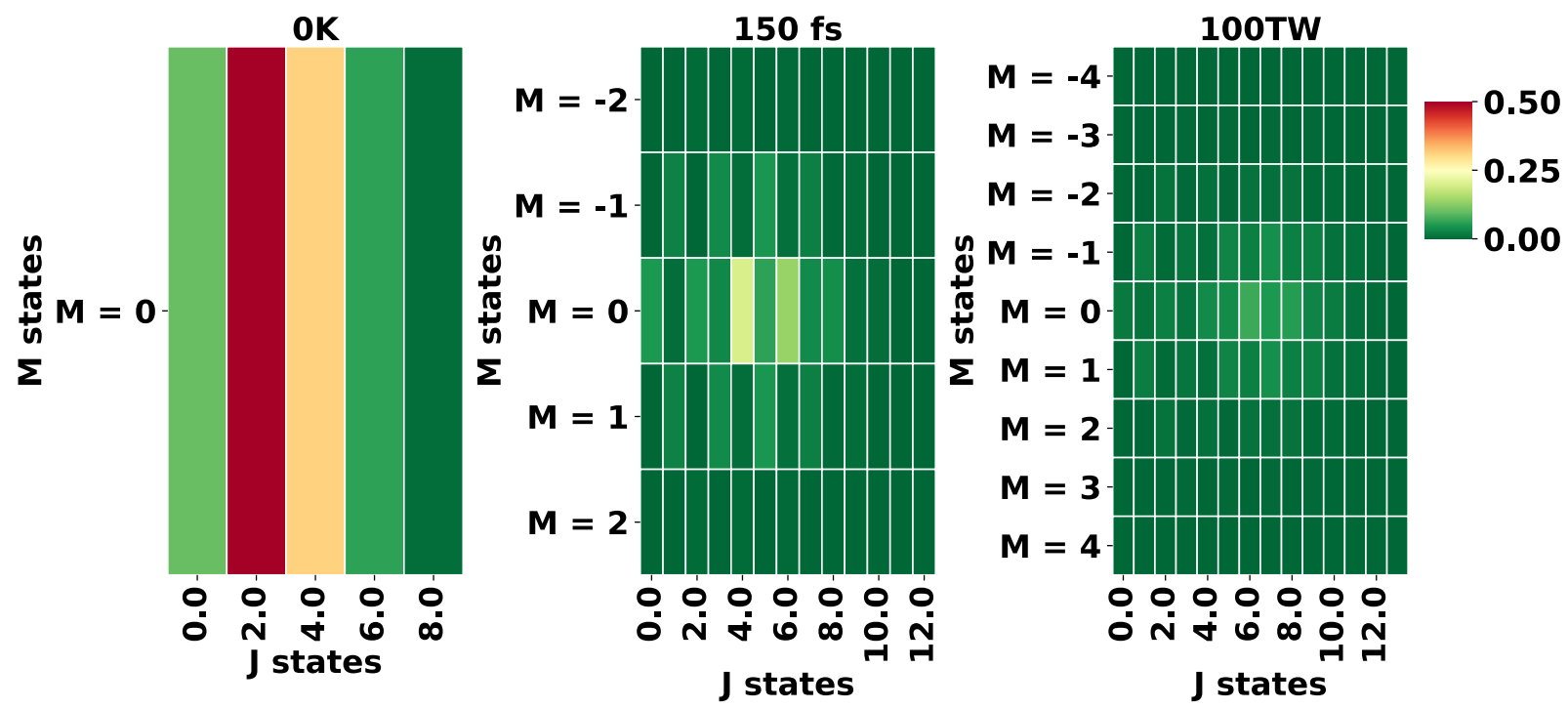

Figure 2. Population of the different rotational state for highest alignment achieved in Fig. 1 with (a) temperature of $0 \mathrm{~K}$ (b) $150 \mathrm{fs}$ FWHM and (c) Intensity of $100 \mathrm{TW} / \mathrm{cm}^{2}$.

whereas, maximum $\left\langle\cos ^{2}(\theta)\right\rangle=0.834$ is observed at FWHM $=150 \mathrm{fs}$, followed by a slight decrease in alignment on increasing FWHM up to $300 \mathrm{fs}$ and again increase in alignment with increase in FWHM up to $700 \mathrm{fs}$. This decrease in alignment could be assigned to the change in the nature of different $J$ state populations, as discussed above.

Before moving to discussion on effect of intensity on LIRA we would like to briefly discuss the ionization probability for the pulse parameters used in this work, as the theoretical model used here does not take into account the photo-ionization. Assuming the ionization potential $\left(I_{p}\right)$ for $\mathrm{CH}_{3} \mathrm{~F}$ to be $12.5 \mathrm{eV}^{39}$, the Keldysh parameter ${ }^{40}\left(\gamma=\sqrt{\frac{I_{p}}{2 \times U_{p}}}, U_{p}=9.337 \times 10^{-5} \times I \times \lambda^{2}\right)$ for intensity of $100 \mathrm{TW} / \mathrm{cm}^{2}, \lambda$ of $800 \mathrm{~nm}$ gives $\gamma=1.023$, which is in the transition regime ${ }^{41}$. Hence ionization is dominated by multi-photon ionization between $1 \mathrm{TW} / \mathrm{cm}^{2}$ and $100 \mathrm{TW} / \mathrm{cm}^{2}$ intensity, however, further increase in intensity will result in $\gamma<1$ and could lead to tunneling ionization of $\mathrm{CH}_{3} \mathrm{~F}$. Nonetheless, in our parametric study, we present results for pulse intensities not larger than $100 \mathrm{TW} / \mathrm{cm}^{2}$. In the simulated results for LIRA with varying pulse intensity we observe that the maximal alignment appears to increase linearly as a function of the intensity (see Fig. 1(c and f)). The analysis of the $J$ states population (see fig. S4 detailed discussion given in SI) indicates an increase in number of excited rotational states with intensity. On increasing the pulse intensity with fixed FWHM, the pulse energy is also increased and consequently, the number of $J$ states populated increases, as well. For increase in intensity from $1 \mathrm{TW} / \mathrm{cm}^{2}$ to $10 \mathrm{TW} / \mathrm{cm}^{2}\left(U_{p}<0.6 \mathrm{eV}\right)$ we find the number of excited rotational states to be the same, however, with slight differences in the populations (see SI Fig. S4). This difference results in increase in the alignment with increase in intensity (see Fig. 1(c and f)). On further increase in intensity from 10 $\mathrm{TW} / \mathrm{cm}^{2}$ to $50 \mathrm{TW} / \mathrm{cm}^{2}\left(U_{p}<3 \mathrm{eV}\right)$, higher $J$ states are excited (see SI Fig. S4 and Fig. 2(c)) and for the $75 \mathrm{TW} / \mathrm{cm}^{2}$ and 100 $\mathrm{TW} / \mathrm{cm}^{2}$ intensities the population of the lower $J$ states $(<4)$ is transferred to higher $J$ states $(J=[4$ to 7$])$. Also, increasing the intensity to $75 \mathrm{TW} / \mathrm{cm}^{2}$ or $100 \mathrm{TW} / \mathrm{cm}^{2}$ causes additional revival patterns to appear in the alignment curves.

\begin{tabular}{|c|c|c|c|c|}
\hline Fig. 3 and 4 & $\begin{array}{c}\text { FWHM } \\
\text { (ps) }\end{array}$ & $\begin{array}{c}\text { Intensity } \\
\left(\mathrm{TW} / \mathrm{cm}^{2}\right)\end{array}$ & $\begin{array}{c}\text { frequency } \\
\text { (THz) }\end{array}$ & $\begin{array}{c}\text { CEP } \\
\text { radian }\end{array}$ \\
\hline (a,e) FWHM & 0.5 to $2^{*}$ & $6 \times 10^{-2}$ & 0.5 & 1.571 \\
(b,f) Intensity & 0.5 & $6 \times 10^{-4}$ to $0.5^{*}$ & 0.9 & 1.571 \\
(c,g) Frequency & 0.5 & $6 \times 10^{-2}$ & 0.1 to $2.0^{*}$ & 1.571 \\
(d,h) CEP & 2 & $6 \times 10^{-2}$ & 0.5 & 0 to 2.355 \\
\hline
\end{tabular}

Table 3. Pulse parameters used to simulate alignment and orientation of $\mathrm{CH}_{3} \mathrm{~F}$ molecule shown in Fig. 3 . CEP of $\pi / 2$ (1.571) radian, peak position at $5 \mathrm{ps}$ and rotational temperature of $2 \mathrm{~K}$. *Varying $\mathrm{THz}$ pulse parameter. 

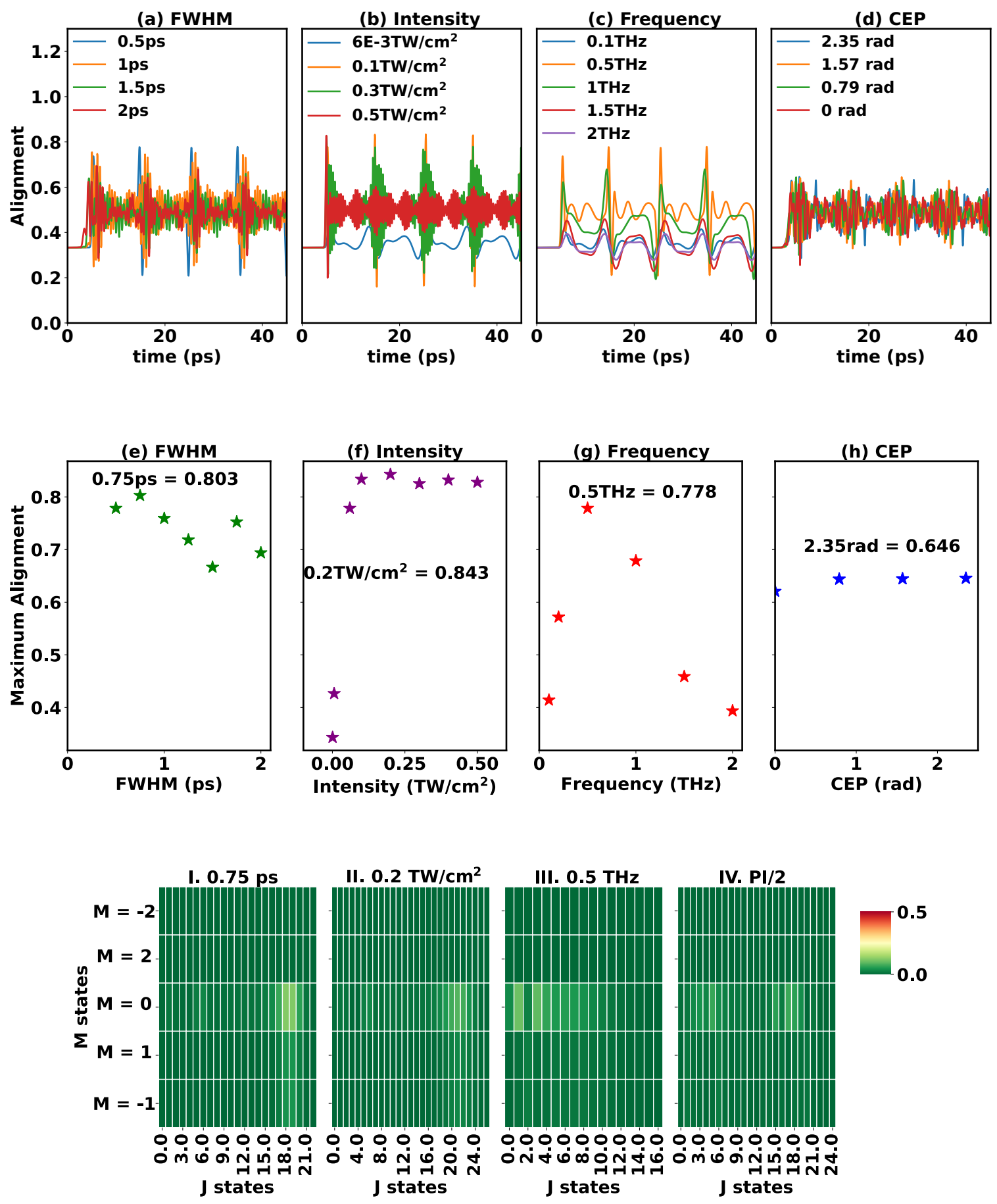

Figure 3. Effect of (a and e) FWHM, (b and f) Intensity (c and g) Frequency and (d and h) CEP on alignment of $\mathrm{CH}_{3} \mathrm{~F}$ with THz pulse. (I-IV) Population of the different rotational state for highest alignment achieved in (e-h) (I) FWHM $=0.75 \mathrm{ps,} \mathrm{(II)}$ Intensity $=0.2 \mathrm{TW} / \mathrm{cm}^{2}$ (III) Frequency $=0.5 \mathrm{THz}$ and (IV) $\mathrm{CEP}=\pi / 2$

Summarizing the results for LIRA (see Fig. 1(d-f)), we observe a monotonous decrease in alignment with increase in temperature whereas, with varying optical pulse, we observe monotonous increase in maximal alignment with intensity. However, non-monotonous behaviour observed with increase in pulse duration. In case of the excitation with an $800 \mathrm{~nm}$ pulse, the interaction with the dipole moment vanishes on the rotational timescale, resulting in molecular alignment only, however, in 

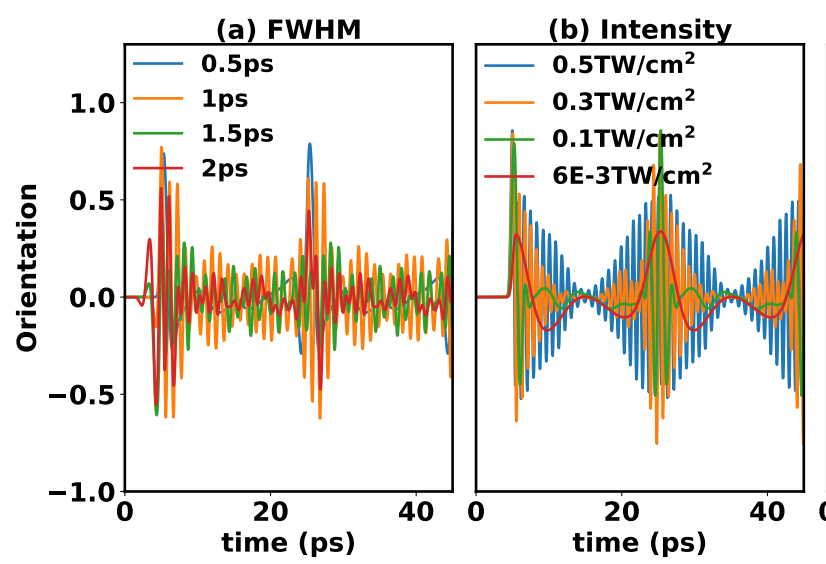

(c) Frequency

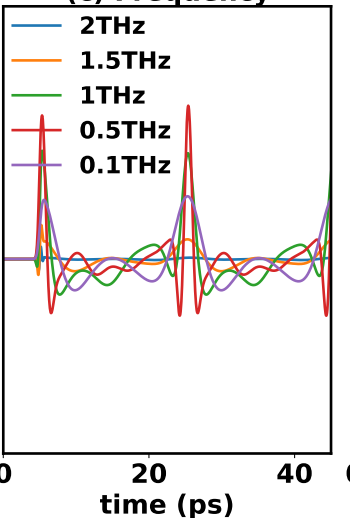

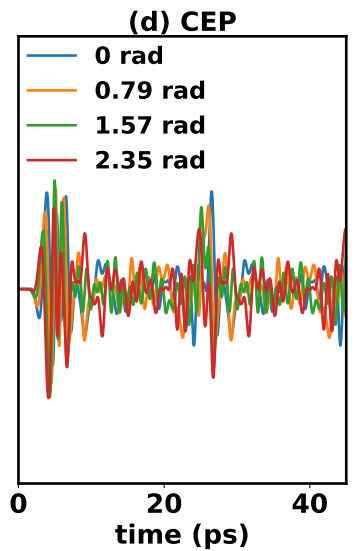
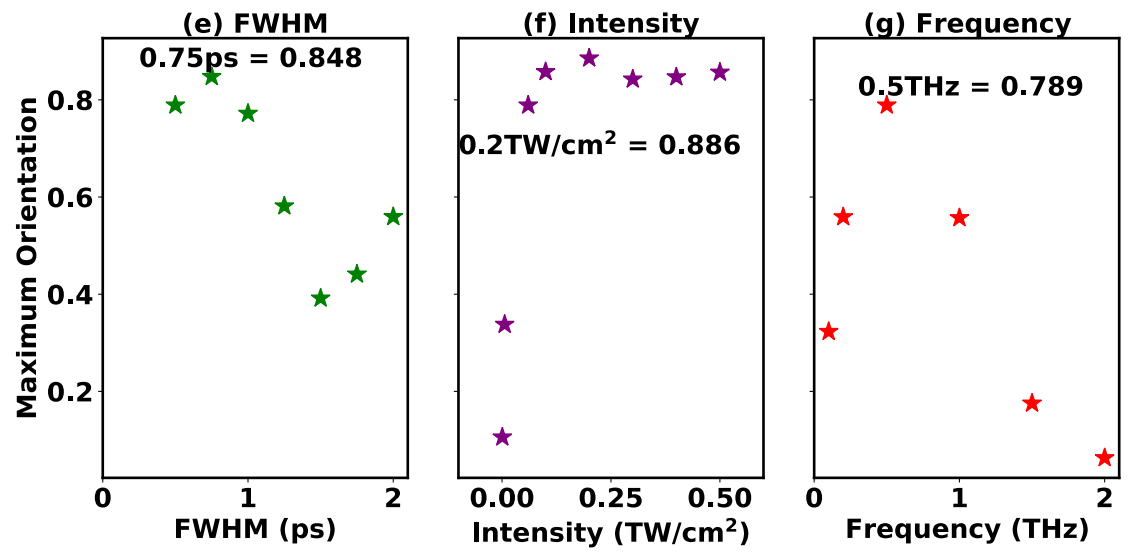

(h) CEP

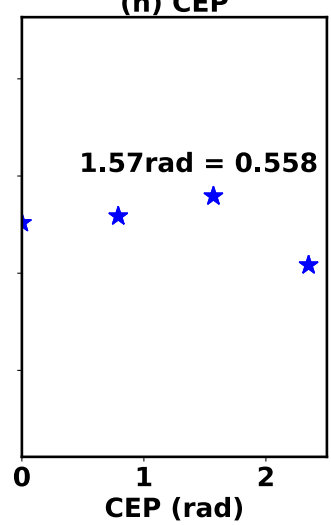

Figure 4. Effect of (a and d) FWHM, (b and e) Intensity (c and f) Frequency and (d and g) CEP on orientation of $\mathrm{CH}_{3} \mathrm{~F}$ with THz pulse.

order to observe molecular orientation, the rotational wave packet needs to be created by light-matter interaction through the permanent dipole and/or hyperpolarizability of molecules. ${ }^{42}$

\section{Effect of Varying THz Pulse Parameters}

In this subsection we will focus on the molecular $\mathrm{AnO}$ with $\mathrm{THz}$ pulses. In addition to field-free molecular $\mathrm{AnO}$ on interaction with the THz pulse, the molecular system could also follows an adiabatic behaviour with the amplitude of the external laser field, which is varying slowly on a time scale comparable to the period of molecular rotation. In this adiabatic process the ensemble of aligned or oriented molecules can be represented using the eigenstates of the field-dressed rotational Hamiltonian. However, in this work we restrict our discussion to non-adiabatic behaviour only. The THz pulse parameters used in further discussion are summarized in Table. 3 .

First, we investigated the role of the THz pulse length, for all FWHM values investigated in this work we observe field-free $\mathrm{AnO}$, however, upon increasing the FWHM of the THz pulse to 2 ps we begin to see the transition from sudden to adiabatic $\mathrm{AnO}$ (see Fig. 3(a, e)). Note that, to achieve complete adiabatic AnO one must use a pulse duration larger than $10 \mathrm{ps.} \mathrm{The}$ alignment curves in Fig. 3(a) show that the FWHM= 0.5 ps results in conventional alignment dynamics, while the FWHM $\geq 1.0$ ps cases show anomalous behaviour with rapid oscillations between the revivals. The orientation dynamics in Fig. 4(a) shows more clear revivals and oscillations. Both maximum alignment and orientation are observed to follow similar trend when varying the FWHM (see Fig. 3(e) and Fig. 4(e)), where highest $\left\langle\cos ^{2}(\theta)\right\rangle=0.803$, and $\langle\cos (\theta)\rangle=0.848$ are observed for $\mathrm{FWHM}=0.75 \mathrm{ps}$. The analysis of the rotational state populations (see Fig. 3(I) details given in SI) shows that the nature of populating the excited rotational states changes at the FWHM values showing a local minimum or maximum in the maximum alignment and orientation curves (see Fig. 3(e) and Fig. 4(e)). Higher maximum AnO values are observed with increase in 
population of higher $J$ states. Additionally, the rapid oscillations in the anomalous alignment and orientation curves are assigned to $J \leftrightarrow J+2$ and $J \leftrightarrow J+1$ beatings, respectively, occurring between high-lying $J$ states.

The effect of varying THz pulse intensity on $\mathrm{AnO}$ is plotted in Fig. 3(b) and Fig. 4(b) where, an anomalous behavior in AnO revivals for intensities $>6 \cdot 10^{-3} \mathrm{TW} / \mathrm{cm}^{2}$ is observed. The maximum AnO values shown in Fig. 3(f) and Fig. 4(f) reveal a monotonous increase in $\mathrm{AnO}$ with $\mathrm{THz}$ pulse intensity up to $0.2 \mathrm{TW} / \mathrm{cm}^{2}$ and achieve highest $\left\langle\cos ^{2}(\theta)\right\rangle=0.843$ (see Fig. 3 (f)) and $\langle\cos (\theta)\rangle=0.886$ (see Fig. 4 (f)). However, further increase in intensity leads to small dips in the maximal AnO at an intensity of $0.3 \mathrm{TW} / \mathrm{cm}^{2}$. The analysis of PDs (given in Fig. 4(II) and in SI) leads us to understand that with increase in THz pulse intensity a broad range of $J$ space is excited (in this case up to $|J=29, M=0\rangle$ state). This broad excitation for intensities between $0.3 \mathrm{TW} / \mathrm{cm}^{2}$ and $0.5 \mathrm{TW} / \mathrm{cm}^{2}$ leads to rapid oscillations in the AnO curves, which are found to originate from the $J \leftrightarrow J+2$ and $J \leftrightarrow J+1$ beatings for higher $J$ states.

On varying the THz pulse carrier frequency (see Fig. 3 (c, g) and Fig. 4 (c, g)), we observe conventional AnO curves. On changing the central pulse frequency, the photon energy changes, and for attaining resonance with a particular rotational excitation, the frequency has to be tuned suitably. The value of maximum achieved AnO is small for both low (0.1 THz) and high (1.5-2.0 THz) frequencies. At low frequencies the field is off resonant with higher $J$ transitions, while at high frequencies the lower $J$ transitions (see SI for more details and PD plots). However, for a pulse with frequency of $0.5 \mathrm{THz}$ (see Fig. 3(III) for PD) a relatively more pronounced resonance between the rotational transitions and pulse frequency leads to highest $\left\langle\cos ^{2}(\theta)\right\rangle=$ 0.778 and $\langle\cos (\theta)\rangle=0.789$. Additionally, on investigating the effect of changing the CEP between 0 and $3 \pi / 4$ for THz pulses leading to anomalous AnO (see pulse parameters in Table 3) we observe that the AnO revival plots change with changing the $\mathrm{CEP}$. On the other hand, the maximum AnO values appear to show very small change on varying the CEP values in between 0 to $3 \pi / 4$. Maximum $\left\langle\cos ^{2}(\theta)\right\rangle=0.646$ and $\langle\cos (\theta)\rangle=0.558$ is achieved at CEP of $3 \pi / 4$ and $\pi / 2$ respectively. The PD plots for for different CEP values, with CEP $=\pi / 2$ shown in Fig. 3(IV) and the rest given in the SI, do not show any significant difference for varying CEP.

\section{Summary and Conclusions}

In this work we investigated the laser-induced alignment and orientation (AnO) dynamics of the $\mathrm{CH}_{3} \mathrm{~F}$ molecule for nonresonant optical and intense few-cycle $\mathrm{THz}$ pulses. By analyzing the interplay between laser pulse parameters and the resulting rotational population distributions, the physics underlying behind specific AnO dynamics was revealed. Also, specific pulse parameter values for high values of laser-induced alignment and orientation for the $\mathrm{CH}_{3} \mathrm{~F}$ molecule could be predicted. For both the optical and THz pulses we identified two types of AnO dynamics, conventional where rotational revivals are distinctly observed and anomalous where, in between half and full revival several oscillations appear. Detailed analysis of the population distributions (PD) given in the SI, which shows that the anomalous AnO behaviour was identified to originate from large portions of populations in highly-excited rotational states (large $J$ values), either localized to a few higher states (large $M$ value), or showing a broad distribution in $J$ space.

In the non-resonant optical pulse case we observe a monotonous decrease in alignment with increase in temperature, and for the pulse parameters investigated, we observe that increasing the intensity increases the maximal alignment, but the maximal alignment is not a monotonous function of the pulse duration. For the longest pulses investigated, the alignment curves become anomalous. For the $800 \mathrm{~nm}$ pulse at $2 \mathrm{~K}$ the maximum alignment achieved is $\left\langle\cos ^{2}(\theta)\right\rangle=0.834$ for $\mathrm{CH}_{3} \mathrm{~F}$, using FWHM of $150 \mathrm{fs}$ and intensity of $100 \mathrm{TW} / \mathrm{cm}^{2}$. As for the $\mathrm{THz}$ pulse case, both the maximal alignment and orientation show a non-monotonous dependence on the FWHM, intensity and carrier frequency of the THz pulse. Nonetheless, the specific dynamics can be explained by the pulse properties and the resulting rotational population distributions. Considering the Fourier transform of the THz pulses, i.e., their spectral properties is a key tool in the analysis. The carrier envelope phase (CEP) dependence of the AnO dynamics showed that $\mathrm{AnO}$ are both sensitive to the CEP, but the maximal AnO is not significantly altered. Maximum $\mathrm{AnO}$ for $\mathrm{CH}_{3} \mathrm{~F}$ at $2 \mathrm{~K}$ is achieved with $\mathrm{THz}$ pulse parameters of $\mathrm{FWHM}=0.75 \mathrm{ps}$, Intensity $=0.2 \mathrm{TW} / \mathrm{cm}^{2}$ and frequency $=0.5 \mathrm{THz}$.

Overall, our work systematically explores optical and THz-pulse induced alignment and orientation as a function of both intrinsic molecular parameters (polarizability, rotational constants and nuclear spin statistics, as obtained from quantum chemistry simulations) and experimental parameters (laser intensity, pulse duration, frequency, CEP, and temperature of the molecular sample), with particular attention towards parameters that reflect typical conditions in the laboratory. The improved state-of-the-art molecular beams produced by supersonic expansions through valves of the Even Lavie type helps in achieving rotational temperature as low as $2 \mathrm{~K}^{43}$ and hence, presents an unique opportunity to achieve high alignment for symmetric top molecules with non-resonating single pulse, and for exploring the fundamental of excitation dynamics. Control over 
oriented higher angular momentum states in a preferential direction by means of laser-induced rotational excitation is the first crucial step towards manipulating the molecular-axis distribution, thereby manipulating further molecular processes. Our approach of identifying suitable parametric regime to obtain a large degree of orientation and alignment, as observed for $\mathrm{CH}_{3} \mathrm{~F}$, can provide an experimental basis and potential complimentary support for advanced experimental design in atto and femto-chemistry, giving new insights in the understanding of AnO dynamics in molecules. The prescription can be extended for other heteronuclear molecules as well, to understand different aspects of laser-induced unidirectional rotation, and possible further ultrafast reaction dynamics or chemical separation experiments in the molecular frame.

\section{Methods}

All the molecular parameters for $\mathrm{CH}_{3} \mathrm{~F}$ were calculated with the coupled cluster theory considering single, double, and perturbative triple excitations CCSD $(\mathrm{T})^{44-46}$ and the aug-cc-pVDZ basis set ${ }^{47}$, as implemented in the ORCA 4.1 package $^{48,49}$. Further AnO dynamics of $\mathrm{CH}_{3} \mathrm{~F}$ molecules with $800 \mathrm{~nm}$ to $\mathrm{THz}$ pulse were calculated by solving the time-dependent Schrödinger equation using the LIMAO package ${ }^{50}$. In LIMAO the time-dependent Schrödinger equation

$$
\mathrm{i} \hbar \partial_{t}|\Psi(t)\rangle=\hat{H}(t)|\Psi(t)\rangle=\left(\hat{H}_{\mathrm{mol}}+\hat{H}_{\mathrm{ind}}(t)\right)|\Psi(t)\rangle
$$

is solved with the Hamiltonian given as the sum of a field-free rigid rotor molecular Hamiltonian $\left(\hat{H}_{\text {mol }}\right)$, and an interaction term $\left(\hat{H}_{\text {ind }}(t)\right)$.

The interaction term with the external light field is given by

$$
\hat{H}_{\text {ind }}(t)=-\mu \varepsilon(t)-\frac{1}{2} \varepsilon(t)(\alpha \varepsilon(t))=\hat{V}_{\text {dip }}(t)+\hat{V}_{\text {pol }}(t)
$$

where $\mu$ is the permanent electric dipole moment and $\alpha$ is the polarizability tensor. In Eq. (2) $\hat{V}_{\text {dip }}(t)$ term is the interaction of light with the dipole moment of the molecule, and $\hat{V}_{\mathrm{pol}}(t)$ is the interaction with the polarizability. The electric fields of light pulses linearly polarized along the $Z$-axis of the laboratory-fixed (LF) frame are assumed hereafter. For gaussian pulse shape with a single central frequency, $\omega$, the $Z$ component of the electric field is given as

$$
\varepsilon_{Z}(t)=\varepsilon_{\omega}(t) \cos \left(\omega t+\phi_{\omega}\right)
$$

where $\varepsilon_{\omega}(\mathrm{t})$ is the envelope function and $\phi_{\omega}$ is the carrier envelope phase (CEP). In case of an intense laser pulse with $\omega$ in the visible and near infrared frequency regions, its interaction with the $\mu$ dipole of molecules vanishes after time averaging, therefore, the interaction is primarily that with $\alpha$ of molecules. However, for $\mathrm{THz}$ pulses the field-dipole interaction is dominant. The time-dependent degree of AnO of a symmetric top molecule can be computed as the expectation values $\left\langle\cos ^{2}(\theta)\right\rangle(t)=\left\langle\Psi(t)\left|\cos ^{2}(\theta)\right| \Psi(t)\right\rangle$ and $\langle\cos (\theta)\rangle(t)=\langle\Psi(t)|\cos (\theta)| \Psi(t)\rangle$, respectively, where $\theta$ is the angle between the molecular symmetry axis and the lab-fixed $Z$ axis. The rotational wave packet is expressed as,

$$
|\Psi(t)\rangle=\sum_{J, K, M} C_{J, K, M}(t)|J K M\rangle,
$$

where, $|J K M\rangle$ are the symmetric top rotational eigenstates.

Population of $i^{\text {th }}$ rotational state is calculated using Eq. 5:

$$
P_{i}=\frac{e^{-\frac{E_{i}}{k T}}}{\sum_{l} e^{-\frac{E_{l}}{k T}}}
$$

where $\mathrm{k}$ is the Boltzmann constant and $E_{i}$ is the energy of the $i^{\text {th }}$ rotational state.

\section{Data Availability}

The datasets used and/or analysed during the current study available from the corresponding author on reasonable request.

\section{Acknowledgements}

ELI-ALPS is supported by the European Union and co-financed by the European Regional Development Fund (GI-NOP-2.3.615-2015-00001). KC and MUK acknowledge Project no. 2019-2.1.13-TÉT-IN-2020-00059 which has been implemented with the support provided from the National Research, Development and Innovation Fund of Hungary, financed under the 
2019-2.1.13-TÉT-IN funding scheme; and funding from PaNOSC European project. This research was supported by the János Bolyai Research Scholarship of the Hungarian Academy of Sciences, awarded to TSz, and by the ÚNKP-20-5 and ÚNKP-20-3 New National Excellence Program of the Ministry for Innovation and Technology from the source of the National Research, Development and Innovation Fund, awarded to TSz and IS, respectively. The Hungarian co-authors are also grateful to NKFIH for additional support (Grant No. FK134291).

\section{Author contributions statement}

M.U.K. and T.S. conceived the scientific idea and supervised the work. K.C., I.S. and T.S. did calculations. All authors analysed the data and reviewed the manuscript.

\section{Ethics declarations}

\section{Conflicts of interest}

There are no conflicts to declare.

\section{References}

1. Holmegaard, L. et al. Photoelectron angular distributions from strong-field ionization of oriented molecules. Nat. Phys. 6, 428-432 (2010).

2. Spence, J. C. H. \& Doak, R. B. Single molecule diffraction. Phys. Rev. Lett. 92, 198102, DOI: 10.1103/PhysRevLett.92. 198102 (2004).

3. Torres, R., De Nalda, R. \& Marangos, J. Dynamics of laser-induced molecular alignment in the impulsive and adiabatic regimes: A direct comparison. Phys. Rev. A 72, 023420 (2005).

4. Sándor, P. et al. Angle-dependent strong-field ionization of halomethanes. The J. chemical physics 151, 194308 (2019).

5. Hay, N. et al. High-order harmonic generation in laser-aligned molecules. Phys. Rev. A 65, 053805 (2002).

6. Litvinyuk, I. V. et al. Alignment-dependent strong field ionization of molecules. Phys. review letters 90, 233003 (2003).

7. Li, R.-J. et al. Rotational alignment of product molecules from the reactions sr+ ch3br, c2h5br, n-c 3h7br, i-c 3h7br by means of plif. Chem. physics letters 220, 281-285 (1994).

8. Imanbaew, D. et al. Pump-probe fragmentation action spectroscopy: A powerful tool to unravel light-induced processes in molecular photocatalysts. Angewandte Chemie 129, 5563-5566 (2017).

9. Jin, C., Wang, S.-J., Zhao, X., Zhao, S.-F. \& Lin, C. Shaping attosecond pulses by controlling the minima in high-order harmonic generation through alignment of co 2 molecules. Phys. Rev. A 101, 013429 (2020).

10. Vozzi, C. et al. Generalized molecular orbital tomography. Nat. Phys. 7, 822-826 (2011).

11. Stapelfeldt, H. \& Seideman, T. Colloquium: Aligning molecules with strong laser pulses. Rev. Mod. Phys. 75, 543 (2003).

12. Shapiro, M. \& Brumer, P. Principles of the quantum control of molecular processes (Wiley VCH, 2003).

13. Koch, C. P., Lemeshko, M. \& Sugny, D. Quantum control of molecular rotation. Rev. Mod. Phys. 91, 035005 (2019).

14. Ortigoso, J., Rodriguez, M., Gupta, M. \& Friedrich, B. Time evolution of pendular states created by the interaction of molecular polarizability with a pulsed nonresonant laser field. The J. chemical physics 110, 3870-3875 (1999).

15. Seideman, T. Revival structure of aligned rotational wave packets. Phys. Rev. Lett. 83, 4971 (1999).

16. Holmegaard, L. et al. Control of rotational wave-packet dynamics in asymmetric top molecules. Phys. Rev. A 75, 051403 (2007).

17. Peng, P., Bai, Y., Li, N. \& Liu, P. Measurement of field-free molecular alignment by balanced weak field polarization technique. AIP Adv. 5, 127205 (2015).

18. Bert, J. et al. Optical imaging of coherent molecular rotors. Laser \& Photonics Rev. 14, 1900344 (2020).

19. Mizuse, K., Sakamoto, N., Fujimoto, R. \& Ohshima, Y. Direct imaging of direction-controlled molecular rotational wave packets created by a polarization-skewed double-pulse. Phys. Chem. Chem. Phys. 22, 10853-10862 (2020).

20. Liu, J.-S., Cheng, Q.-Y., Yue, D.-G., Zhou, X.-C. \& Meng, Q.-T. Influence factor analysis of field-free molecular orientation. Chin. Phys. B 27, 033301 (2018). 
21. Fleischer, S., Zhou, Y., Field, R. W. \& Nelson, K. A. Molecular orientation and alignment by intense single-cycle thz pulses. Phys. Rev. Lett. 107, 163603, DOI: 10.1103/PhysRevLett.107.163603 (2011).

22. Ortigoso, J. Conservation of molecular alignment for cyclic rotational wave packets in periodic pulse trains. Phys. Rev. Lett. 93, 073001, DOI: 10.1103/PhysRevLett.93.073001 (2004).

23. Fleischer, S., Averbukh, I. S. \& Prior, Y. Isotope-selective laser molecular alignment. Phys. Rev. A 74, 041403 (2006).

24. Hertz, E., Rouzée, A., Guérin, S., Lavorel, B. \& Faucher, O. Optimization of field-free molecular alignment by phase-shaped laser pulses. Phys. Rev. A 75, 031403 (2007).

25. Baek, D., Hasegawa, H. \& Ohshima, Y. Unveiling the nonadiabatic rotational excitation process in a symmetric-top molecule induced by two intense laser pulses. The J. chemical physics 134, 224302 (2011).

26. Hasegawa, H. \& Ohshima, Y. Decoding the state distribution in a nonadiabatic rotational excitation by a nonresonant intense laser field. Phys. Rev. A 74, 061401 (2006).

27. Kühn, S. et al. The eli-alps facility: the next generation of attosecond sources. J. Phys. B: At. Mol. Opt. Phys. 50, 132002 (2017).

28. Budriūnas, R. et al. $53 \mathrm{w}$ average power cep-stabilized opcpa system delivering $5.5 \mathrm{tw}$ few cycle pulses at $1 \mathrm{khz}$ repetition rate. Opt. express 25, 5797-5806 (2017).

29. Gupta, A. et al. Nonlinear thz spectroscopy user facility at eli-alps. In 2020 45th International Conference on Infrared, Millimeter, and Terahertz Waves (IRMMW-THz), 1-2 (IEEE, 2020).

30. Liao, G.-Q. et al. Towards terawatt-scale spectrally tunable terahertz pulses via relativistic laser-foil interactions. Phys. Rev. X 10, 031062, DOI: 10.1103/PhysRevX.10.031062 (2020).

31. Papousek, D. et al. Far infrared spectrum and ground state parameters of 12ch3f. J. Mol. Spectrosc. 159, 33-41 (1993).

32. Shimanouchi, T., Matsuura, H., Ogawa, Y. \& Harada, I. Tables of molecular vibrational frequencies part 10. J. Phys. Chem. Ref. Data 9, 1149-1254 (1980).

33. Simkó, I., Chordiya, K., Császár, A. G., Kahaly, M. U. \& Szidarovszky, T. A quantum-chemical perspective on the laser-induced alignment and orientation dynamics of the $\mathrm{ch}_{3} \mathrm{x}(\mathrm{x}=\mathrm{f}, \mathrm{cl}, \mathrm{br}, \mathrm{i})$ molecules (2021). 2109.08413.

34. Bunker, P. R. \& Jensen, P. Molecular symmetry and spectroscopy (NRC Research Press, 2006).

35. Xu, N. et al. Field-free alignment of molecules at room temperature. Opt. express 14, 4992-4997 (2006).

36. Even, U., Jortner, J., Noy, D., Lavie, N. \& Cossart-Magos, C. Cooling of large molecules below $1 \mathrm{k}$ and he clusters formation. The J. Chem. Phys. 112, 8068-8071 (2000).

37. Hagena, O. \& Obert, W. Cluster formation in expanding supersonic jets: Effect of pressure, temperature, nozzle size, and test gas. The J. Chem. Phys. 56, 1793-1802 (1972).

38. Harms, J., Toennies, J. P. \& Dalfovo, F. Density of superfluid helium droplets. Phys. Rev. B 58, 3341 (1998).

39. Moxom, J. et al. Fragmentation and ionization of ch3f by positron and electron impact. Nucl. Instruments Methods Phys. Res. Sect. B: Beam Interactions with Mater. Atoms 143, 112-120 (1998).

40. Keldysh, L. et al. Ionization in the field of a strong electromagnetic wave. Sov. Phys. JETP 20, 1307-1314 (1965).

41. Pan, C.-L., Lin, C.-H., Yang, C.-S. \& Zaytsev, A. Laser ablation of polymethylmethacrylate (pmma) by phase-controlled femtosecond two-color synthesized waveforms. In Chapter, vol. 15, 335-358 (IntechOpen, 2016).

42. Oda, K., Hita, M., Minemoto, S. \& Sakai, H. All-optical molecular orientation. Phys. review letters 104, 213901 (2010).

43. Grzesiak, J. et al. Production of rotationally cold methyl radicals in pulsed supersonic beams. Rev. Sci. Instruments 89, 113103 (2018).

44. Noga, J. \& Bartlett, R. J. The full ccsdt model for molecular electronic structure. The J. chemical physics 86, 7041-7050 (1987).

45. Raghavachari, K., Trucks, G. W., Pople, J. A. \& Head-Gordon, M. A fifth-order perturbation comparison of electron correlation theories. Chem. Phys. Lett. 157, 479-483 (1989).

46. Watts, J. D. \& Bartlett, R. J. The coupled-cluster single, double, and triple excitation model for open-shell single reference functions. The J. chemical physics 93, 6104-6105 (1990).

47. Dunning Jr, T. H. Gaussian basis sets for use in correlated molecular calculations. i. the atoms boron through neon and hydrogen. The J. chemical physics 90, 1007-1023 (1989). 
48. Neese, F. The orca program system. Wiley Interdiscip. Rev. Comput. Mol. Sci. 2, 73-78 (2012).

49. Neese, F. Software update: the orca program system, version 4.0. Wiley Interdiscip. Rev. Comput. Mol. Sci. 8, e1327 (2018).

50. Szidarovszky, T., Jono, M. \& Yamanouchi, K. Limao: Cross-platform software for simulating laser-induced alignment and orientation dynamics of linear-, symmetric-and asymmetric tops. Comput. Phys. Commun. 228, 219-228 (2018). 


\section{Supplementary Files}

This is a list of supplementary files associated with this preprint. Click to download.

- CH3FAnOSI.pdf 